\title{
Diagnostic and prognostic evaluation of fluorodeoxyglucose positron emission tomography/ computed tomography and its correlation with serum cancer antigen-125 (CA125) in a large cohort of ovarian cancer patients
}

\author{
Laura Evangelista ${ }^{1}$, Maurizia Dalla Palma², Michele Gregianin ${ }^{1}$, Margherita Nardin $^{3}$, Anna Roma ${ }^{2}$, \\ Maria Ornella Nicoletto ${ }^{2}$, Giovanni Battista Nardelli ${ }^{4}$, Vittorina Zagonel ${ }^{2}$ \\ ${ }^{\prime}$ Radiotherapy and Nuclear Medicine Unit, Istituto Oncologico Veneto IOV - IRCCS, Padova, Italy \\ ${ }^{2}$ Medical Oncology Unit, Istituto Oncologico Veneto IOV - IRCCS, Padova, Italy \\ ${ }^{3}$ Oncological Radiology Unit, Istituto Oncologico Veneto IOV - IRCCS, Padova, Italy \\ ${ }^{4}$ Department of Gynecological Sciences and Human Reproduction, University of Padova, Padova, Italy
}

\section{Abstract}

Objective: We evaluated the efficacy of 18F-fluorodeoxyglucose (FDG) positron emission tomography/computed tomography (PET/CT) in recurrent disease, response to therapy, and long-term follow-up of ovarian cancer (OC) patients in relation to cancer antigen-125 (CA125) levels and the prognostic meaning of this modality in this subset of subjects.

Material and Methods: Between 2005 and 2015, we retrospectively evaluated 125 patients affected by OC who underwent FDG PET/CT imaging at our institution. The indications for PET/CT were recurrence of disease in 78 patients, therapy response assessment in 29 , and follow-up in 18. The results of FDG PET/CT were compared with those of histopathology and clinical and radiological progression during follow-up for at least 6 months. The median long-term follow-up was 33 months. The diagnostic accuracies for the different clinical settings were evaluated. The relationships among global survival (GS), FDG PET/CT results, and CA125 levels were evaluated by both Kaplan-Meier and Cox regression analysis. Results: CA1 25 results were positive (>35 UI/mL) in 62 patients and negative in 63 (49\% vs. 51\%). The sensitivity and specificity of CA125 were $72 \%$ and $91 \%$, respectively. PET/CT imaging showed a sensitivity of $98.6 \%$ and a specificity of $77.8 \%$ for the assessment of recurrent disease, and a sensitivity of $72.7 \%$ and a specificity of $88.9 \%$ for therapy evaluation. Meanwhile, in 18 patients evaluated during follow-up, the specificity was $82.3 \%$. GS was significantly higher in case of negative CA125 values at the time of FDG PET/CT, of a negative PET/CT scan and when no evidence of peritoneum recurrence and distant metastases was determined by PET. Multivariate regression analysis showed that only age and peritoneum recurrence as determined by PET were identified as independent predictors of poor prognosis.

Conclusion: Metabolic imaging with FDG PET/CT proved useful in patients where OC recurrence was suspected, even when the value of tumor marker CA125 was in a normal range. A positive PET/CT scan and the presence of peritoneum recurrence at PET were associated with a poor prognosis after approximately 30 months. (J Turk Ger Gynecol Assoc 2015; 16: 137-44)

Keywords: Ovarian cancer, FDG-PET/CT, CA125, disease-free survival, overall survival.

Received: 26 May, 2015

Accepted: 16 July, 2015

Available Online Date: 06 August, 2015

\section{Introduction}

Ovarian cancer (OC) accounts for approximately $3 \%$ of cancers in women; 125,000 women are estimated to die of the disease each year. OC is curable when identified at an early stage. However, because there is no effective screening test for this tumor and its symptoms are vague, approximately $70-80 \%$ of patients are diagnosed at an advanced stage of the disease (stage III and IV, in accordance with the International
Federation of Gynecology and Obstetrics (FIGO)); this means that the survival rate is low (1). More than $70 \%$ of stage III-IV patients have a relapse of the disease, and even in stage I or II, the relapse rate is $20-25 \%$ (2). The serial evaluation of serum cancer antigen-125 (CA125) can be useful to non-invasively detect the recurrence of disease because an elevation in CA125 levels can determine the presence of disease with a high accuracy of $79 \%$ to $95 \%$, thus preceding a clinically apparent recurrence by 3 to 6 months $(3,4)$. However, normal CA125 levels cannot exclude the presence of recurrent 
Table 1. Characteristics of patients

\begin{tabular}{|c|c|}
\hline \multicolumn{2}{|l|}{ Characteristics } \\
\hline Median age (range), years & $56(18-82)$ \\
\hline \multicolumn{2}{|l|}{ Stage at diagnosis, $\mathrm{n}(\%)$} \\
\hline I & $20(16.0)$ \\
\hline II & $13(10.4)$ \\
\hline III & $72(57.6)$ \\
\hline IV & $9(7.2)$ \\
\hline Unknown & $11(8.8)$ \\
\hline \multicolumn{2}{|l|}{ Histology, n (\%) } \\
\hline Papillary serous adenocarcinoma & $78(62.4)$ \\
\hline Endometroid adenocarcinoma & $11(8.8)$ \\
\hline Mucinous adenocarcinoma & $4(3.2)$ \\
\hline Clear cell carcinoma & $5(4.0)$ \\
\hline Ovarian sex cord tumors & $7(5.6)$ \\
\hline Transitional cell carcinoma & $1(0.8)$ \\
\hline Borderline & $7(5.6)$ \\
\hline Poorly differentiated adenocarcinoma & $1(0.8)$ \\
\hline Ovarian sarcoma & $2(1.6)$ \\
\hline Neuroendocrine & $1(0.8)$ \\
\hline Unknown & $8(6.4)$ \\
\hline \multicolumn{2}{|l|}{ Grade, n (\%) } \\
\hline G1 & $10(8.0)$ \\
\hline G2 & $31(24.8)$ \\
\hline G3 & $69(55.2)$ \\
\hline Unknown & $15(12.0)$ \\
\hline
\end{tabular}

disease. Once an increase in CA125 levels is documented, oncologists or clinicians usually request additional imaging studies to identify the site of recurrence and thus choose the best treatment strategy. In this regard, it has been demonstrated that 18F-fluorodeoxyglucose (FDG) positron emission tomography (PET)/computed tomography (CT) is able to detect the early recurrence of disease, even when CA125 levels are in the normal range or are minimally elevated $(5,6)$. Nevertheless, data concerning the relationship between prognosis and FDG $\mathrm{PET} / \mathrm{CT}$ in this group of patients remain indeterminate. Kurosaki et al. (7) demonstrated that the 2-year survival rate was more favorable in patients with a negative PET/CT scan than in those with a positive finding.

In the present study, we aimed to evaluate 1) the efficacy of FDG PET/CT in the recurrence of disease, response to therapy, and long-term follow-up of OC patients by comparing CA125 levels and 2) the prognostic meaning of this modality in this subset of subjects.

\section{Material and Methods}

Between 2005 and 2015, we retrospectively evaluated 125 patients affected by OC who underwent FDG PET/CT imaging at our institution. The indications for PET/CT were recurrence of disease, therapy response assessment, and follow-up. Clinical, demographic, and imaging information were collected from the patients' charts. According to the institutional guidelines, approval from the local ethical committee was not required (in accordance with the nature of the study). However, all patients gave their written consent for treatments and imaging examination.

\section{FDG-PET/CT imaging and analysis}

Whole-body FDG PET/CT was performed using a dedicated scanner (Biograph 16, Siemens Medical Solutions, Hoffman Estates, Illinois, United States) upgraded with high-definition software. Fasting for at least $6 \mathrm{~h}$ was required before the examination. After the injection of approximately $3 \mathrm{MBq}$ of FDG per kg of body weight, patients rested for a period of approximately $60 \mathrm{~min}$. Emission images from the base of the skull to the mid-thigh were acquired for 2-3 min per bed position. For the assessment of the images, we defined the presence of increased FDG uptake outside from the physiological sites as a positive PET/CT scan. In contrast, the absence of significant FDG uptake outside the heart, mediastinal blood pool, stomach, bowel, bladder, and kidney defined as a negative scan. Finally, semiquantitative analysis was performed for each lesion using the maximum standardized uptake value (SUVmax) by drawing a volume of interest (VOI) around suspected lesion sites.

\section{Gold standard and follow-up data}

Diagnoses were verified by clinical follow-up and imaging studies (chest X-rays, CT, bone scans, abdominal ultrasounds, nuclear magnetic resonance, and FDG PET). The diagnosis of the metastatic disease was made when the presence of new lesions at clinical evaluations and/or from imaging studies was demonstrated. In patients without disease relapse, neither a change in ongoing treatment nor further treatment was performed, and a close follow-up with imaging studies was started.

Follow-up examinations and clinical visits were conducted in the Nuclear Medicine/Oncology Radiology Unit and in the Oncology 1 Unit of our institute, respectively.

Global survival (GS) was defined as the length of time from the date of PET/CT to death from any cause. The mean follow-up period was 33 months (range: 1-110). During this observational period, some patients were treated by surgery $(n=13)$, systemic therapy (chemotherapy or hormonal therapy), or radiotherapy $(\mathrm{n}=49)$.

\section{Statistical analysis}

The performance of FDG PET/CT scans was calculated on the basis of per-patient and per-lesion analysis using the standard method. Continuous data were expressed as mean \pm standard deviation or median (range) and nominal data as number (percentage). The ANOVA test was used for defining the difference among and between subsets of patients. Receiver operating characteristic (ROC) analysis and area under curve (AUC) were computed to define the cutoff value of CA125 for determining a positive PET/CT scan. Kaplan-Meier analysis and log-rank tests were used to compare different parameters 


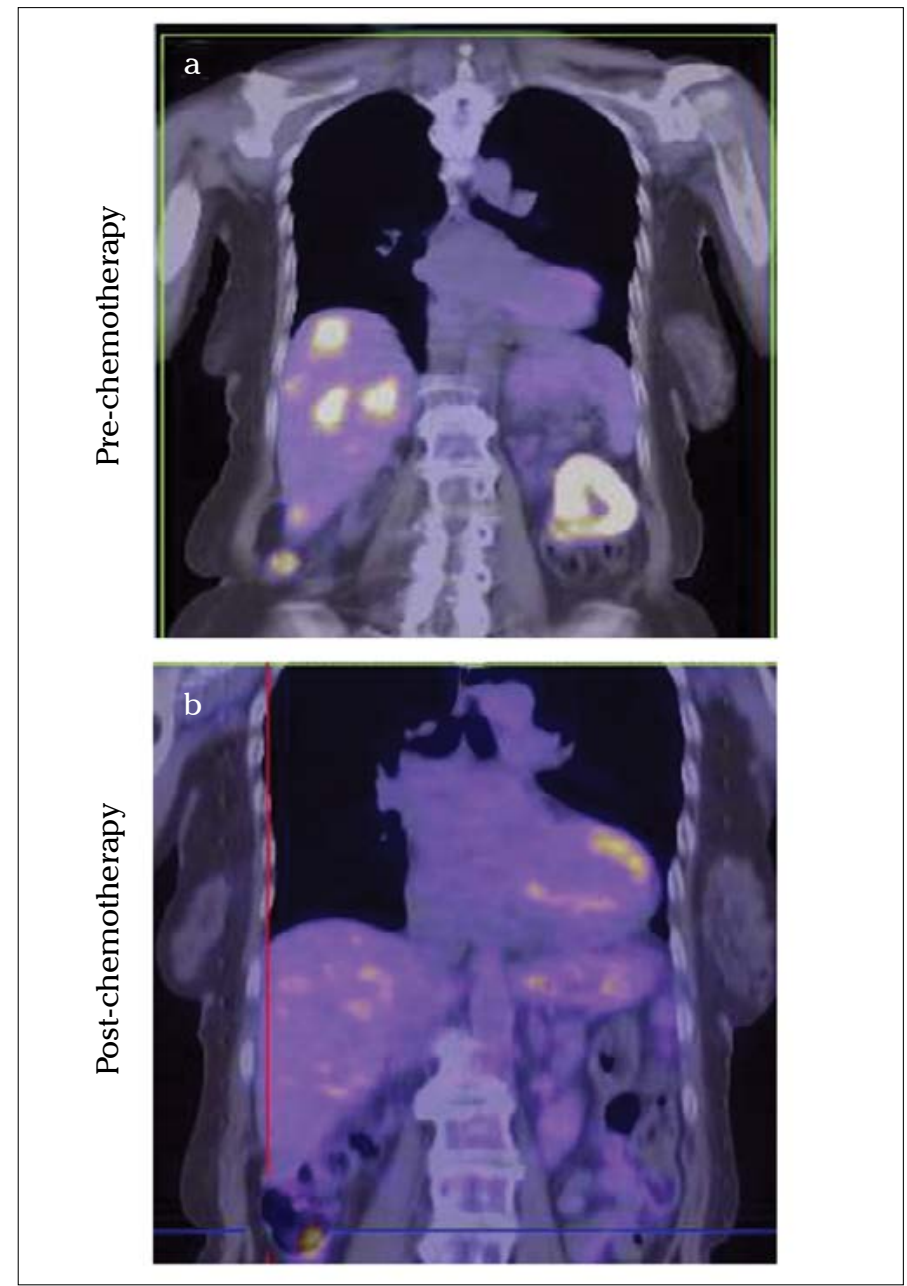

Figure 1. a, b. FDG PET/CT in a 72-year-old woman with recurrent ovarian cancer (FIGO stage IIIC, grade 3 serous papillary ovarian carcinoma, post-hysterectomy, bilateral oophorectomy, and chemotherapy completed 4 years before) presenting with increased CA125 level (150 UI/L) and an uncertain ultrasonography result. Coronal fusion image (a) showed abnormal FDG uptake in the liver and in the left quadrant of the abdomen. The patient underwent 6 cycles of chemotherapy with Caelyx and Oxaliplatin. Seven months later, another FDG PET/CT scan showed the resolution of metabolic activity in the liver and abdomen (b) compatible with a good response to therapy

(e.g., positive vs. negative PET/CT or negative CA125 vs. positive CA125, etc.). Moreover, Cox proportional hazards regression analysis was used to identify independent variables associated with a worse GS. A multivariate analysis was built using variables that showed a value of 0.1 in the univariate model. Statistical analysis was performed using SPSS software for Windows, version 15.0 (SPSS, Chicago, Illinois, United States).

\section{Results}

The characteristics of the patients are reported in Table 1. More than $70 \%$ of the patients were at advanced stages (III-IV according to the FIGO) and had aggressive cancer
Table 2. Accuracies of CA125 and PET/CT in all study populations

\begin{tabular}{|c|c|c|}
\hline & CA125 & FDG PET/CT \\
\hline Sensitivity, CI 95\% & $71.6(61.8-81.4)$ & 95.1 (90.3-99.8) \\
\hline Specificity, CI 95\% & 90.9 (82.4-99.4) & $84.1(73.3-94.9)$ \\
\hline PPV, CI 95\% & 93.5 (88.2-98.9) & $91.7(85.6-97.7)$ \\
\hline NPV, CI 95\% & $63.5(49.3-77.8)$ & $90.2(81.5-99.1)$ \\
\hline Accuracy, CI 95\% & $78.4(71.2-85.6)$ & $91.2(86.2-96.2)$ \\
\hline \multicolumn{3}{|c|}{$\begin{array}{l}\text { PPV: positive predictive value; NPV: negative predictive value; CI: } \\
\text { confidence interval; CA: cancer antigen } \\
\text { FDG PET/CT: fluorodeoxyglucose positron emission tomography/ } \\
\text { computed tomography }\end{array}$} \\
\hline
\end{tabular}
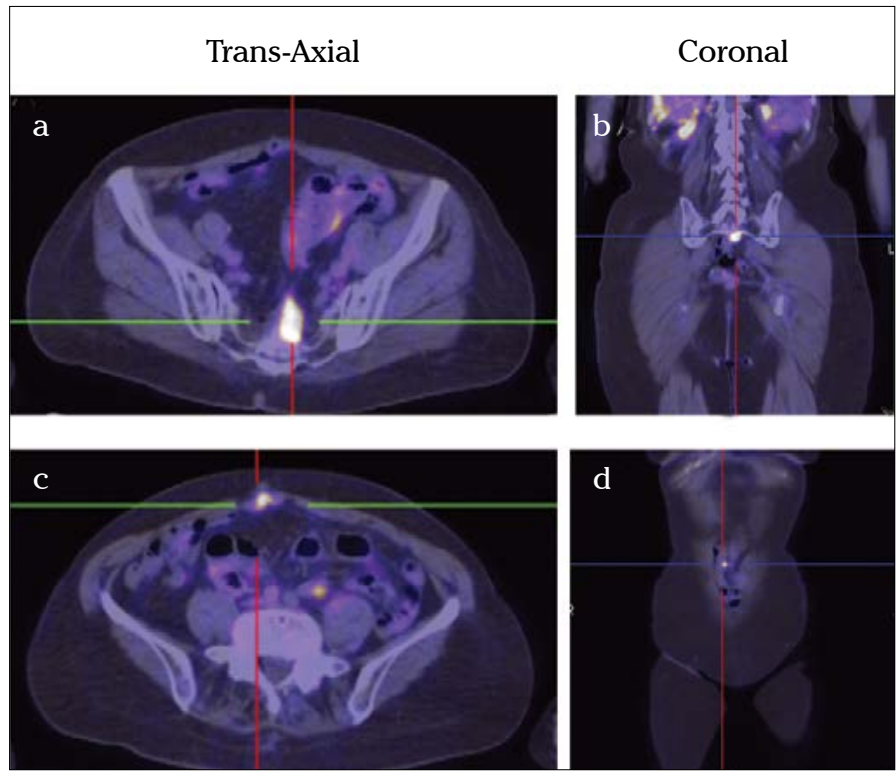

Figure 2. a-d. Axial (a, c) and coronal (b, d) FDG PET/CT in a 45-year-old female with a stage III papillary serous adenocarcinoma with invasive peritoneal implants

(grade $3 ; \mathrm{n}=69$ ). All patients underwent PET/CT, 78 for relapse assessment (Figure 1a, b), 29 for assessment after primary therapy, and 18 for long-term follow-up. The median period between the last chemotherapy and PET/CT used to determine treatment efficacy ( $n=29$ patients) was 4 months (1-8 months). The period between the last treatment and the FDG PET/CT scan was 17 months (1-103). In 81 patients (65\%), the recurrence of disease was pathologically and clinically confirmed. For the evaluation of recurrence, CT was performed on 63 patients, abdominal ultrasonography on 8 , pelvic magnetic resonance imaging on 3, bone scan on 1, and clinical evaluation (both CA125 and histological evaluation) in the remaining 50 patients.

\section{FDG PET/CT and CA125 findings}

The CA125 level at the time of the PET/CT scan was available for all patients (median value: $60 \mathrm{UI} / \mathrm{mL}$; range: 4 to $5777 \mathrm{UI} /$ $\mathrm{mL}$ ): in 62 patients the result was positive (i.e., $>35 \mathrm{UI} / \mathrm{mL}$ ), and in 63 patients the result was negative. The median time between CA125 evaluation and PET/CT was 2 months (1 to 3 
Table 3. Accuracies of CA125 and PET/CT in 96 patients

\begin{tabular}{|l|c|c|c|c|}
\hline \multicolumn{4}{|c|}{ Assessment relapse (n=78) } \\
\hline & CA125 & FDG PET/CT & CA125 & FDG PET/CT \\
\hline Sensitivity, CI 95\% & $73.9(63.5-84.2)$ & $98.6(95.7-100)$ & - & 100 \\
\hline Specificity, CI 95\% & $88.9(68.4-100)$ & $77.8(50.6-100)$ & 100 & $82.3(64.7-99.9)$ \\
\hline PPV, CI 95\% & $98.1(94.8-100)$ & $97.1(93.2-100)$ & - & $25.0(5.0-45.5)$ \\
\hline NPV, CI 95\% & $30.8(6-61)$ & $87.5(65.9-100)$ & $94.4(83.6-100)$ & 100 \\
\hline Accuracy, CI 95\% & $75.6(66.1-85.2)$ & $96.1(91.8-100)$ & $94.4(83.6-100)$ & $83.3(66.1-100)$ \\
\hline $\begin{array}{l}\text { PPV: positive predictive value; NPV: negative predictive value; CI: confidence interval; CA: cancer antigen } \\
\text { FDG PET/CT: fluorodeoxyglucose positron emission tomography/computed tomography }\end{array}$ \\
\hline
\end{tabular}

Table 4. Accuracies of CA125 and PET/CT in 29 patients

\begin{tabular}{|c|c|c|}
\hline & \multicolumn{2}{|c|}{$\begin{array}{c}\text { Assessment relapse after } \\
\text { treatment }(n=29)\end{array}$} \\
\hline & CA125 & FDG PET/CT \\
\hline Sensitivity, CI 95\% & $63.6(35.2-92)$ & $98.6(95.7-100)$ \\
\hline Specificity, CI 95\% & $83.3(66.1-100)$ & $77.8(50.6-100)$ \\
\hline PPV, CI 95\% & 70 (42.9-97) & $97.1(93.2-100)$ \\
\hline NPV, CI 95\% & $78.9(60.1-97.8)$ & $87.5(65.9-100)$ \\
\hline Accuracy, CI 95\% & $75.9(60.3-91.4)$ & $96.1(91.8-100)$ \\
\hline \multicolumn{3}{|c|}{$\begin{array}{l}\text { PPV: positive predictive value; NPV: negative predictive value; CI: } \\
\text { confidence interval; CA: cancer antigen } \\
\text { FDG PET/CT: fluorodeoxyglucose positron emission tomography/ } \\
\text { computed tomography }\end{array}$} \\
\hline
\end{tabular}

months). The CA125 results were true positive in 58 patients (72\%) and true negative in 40 patients (91\%). The sensitivity, specificity, and diagnostic accuracy of the CA125 values were $72 \%, 91 \%$, and $78 \%$, respectively (Table 2). PET/CT yielded a positive finding in 84 patients (67\%); in particular, it gave true positive results in 77 patients (95\%) and false positive results in 7 patients (8\%) (Table 2). In the latter patients, PET/CT showed chronic abdomen inflammation $(n=4)$, bronchitis $(n=2)$, abnormal liver uptake due to the presence of hemangioma $(n=1)$, and adrenal functional adenoma $(n=1)$. The PET/CT result was true negative in 37 patients (84\%) and false negative in 4 patients. Specifically, $\mathrm{PET} / \mathrm{CT}$ missed an instance of liver disease that was detected by CT scan and later histologically confirmed; two micrometastases (diameter $<1 \mathrm{~cm}$ ) of the lung and liver; and two cases of recurrent borderline OC (Figure 2 a-d). The concordance between CA125 and PET/CT demonstrated that 55 patients had positive findings from both methods; however, 29 patients with negative CA1 25 values had positive PET/CT scans. 22 out of these 29 patients had true positive PET findings; thus, PET/CT identified recurrence of disease in $35 \%$ of patients where recurrence was not indicated by CA125 value. The highest accuracy (95\%) was found when $\mathrm{PET} / \mathrm{CT}$ and positive CA125 were associated, although a low negative predictive value was demonstrated (57\%). In patients with a positive PET/CT scan, CA125 values were higher than in those with a negative scan $(284.39 \pm 723.54$ vs.
$66.77 \pm 251.44 \mathrm{UI} / \mathrm{mL}$, respectively), but the results were not statistically significant $(p=0.087)$. A CA125 value of $32.5 \mathrm{U} /$ $\mathrm{mL}$ was considered as an optimal cut-off value for predicting a positive PET/CT scan (AUC 0. 829, $\mathrm{p}<0.001$; sensitivity: $76 \%$ and specificity: $80 \%$ ). The introduction of FDG PET/ CT in the diagnostic flowchart of all 125 patients changed the management in 52 patients (41.6\%), particularly those evaluated for the recurrence of disease $(n=40 / 78 ; 51.2 \%)$.

FDG PET/CT and CA125 findings in 96 patients with relapse Table 3 reports the diagnostic accuracies of CA125 and PET/ CT in patients evaluated for the assessment of relapse and in follow-up. As illustrated, the sensitivities were higher for PET/CT compared to CA125, although the specificities and accuracy were higher for CA125 in both subsets and in the follow-up subset.

FDG PET/CT and CA125 findings in 29 patients with relapse after treatment

By analyzing the diagnostic accuracy of CA125 and PET/CT in 29 patients examined after treatment, FDG PET/CT was more accurate, both in terms of sensitivity and accuracy, than CA 125 (Table 4). However, the specificity of CA 125 was higher than that of PET/CT. The latter result could be due to possible residual inflammation after therapy that can be associated with a higher number of false positive results.

\section{$\mathrm{PET} / \mathrm{CT}$ and lesion site analysis}

52 patients showed suspicious findings by PET/CT for peritoneal carcinomatosis, which was histologically confirmed in 50 of the patients. 43 patients with peritoneal carcinomatosis underwent $\mathrm{PET} / \mathrm{CT}$ for the assessment of relapse, while 7 underwent PET/ CT for the assessment of relapse after treatment. Furthermore, 41 patients had a positive PET/CT result for lymph node disease (15 loco-regional, 13 distant, and 13 loco-regional and distant lymph nodes), in particular 39 subjects evaluated by PET/CT for the assessment of recurrence and 2 subjects evaluated after therapy. PET/CT falsely identified 1 patient with loco-regional lymph node disease and 1 with distant lymph node metastases. Visceral metastases were found by PET/CT in the liver and lungs in 16 and 7 patients, respectively (both liver and lung metastases were found in subjects evaluated for the recurrence of disease, while liver disease was also reported in patients evaluated 

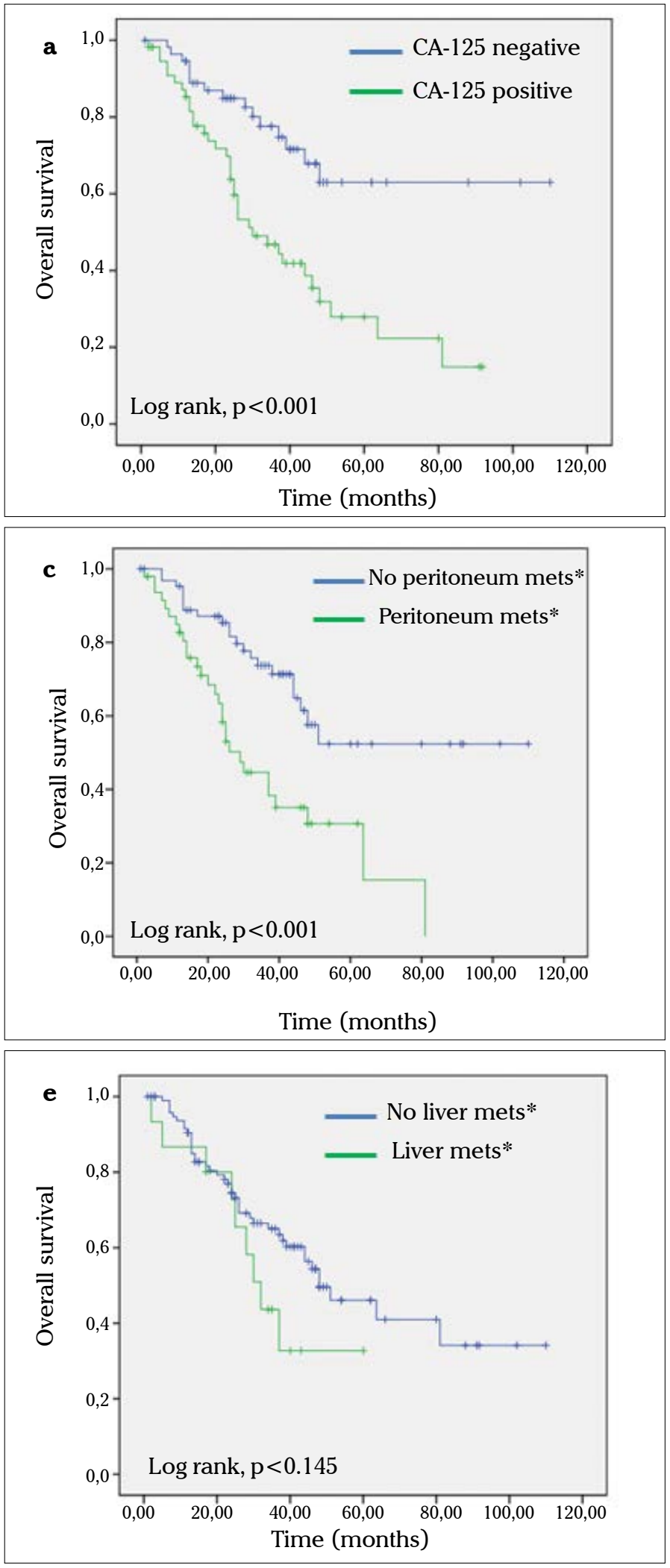

Figure 3. a-f. Overall survival curves based on CA125 results (a), PET/CT findings (b), peritoneal (c), lymph nodal (d), liver (e), and visceral (f) metastases determined by PET/CT images

after therapy). In the latter organ, PET/CT gave a false positive result in one patient. Finally, bone lesions were discovered in 4
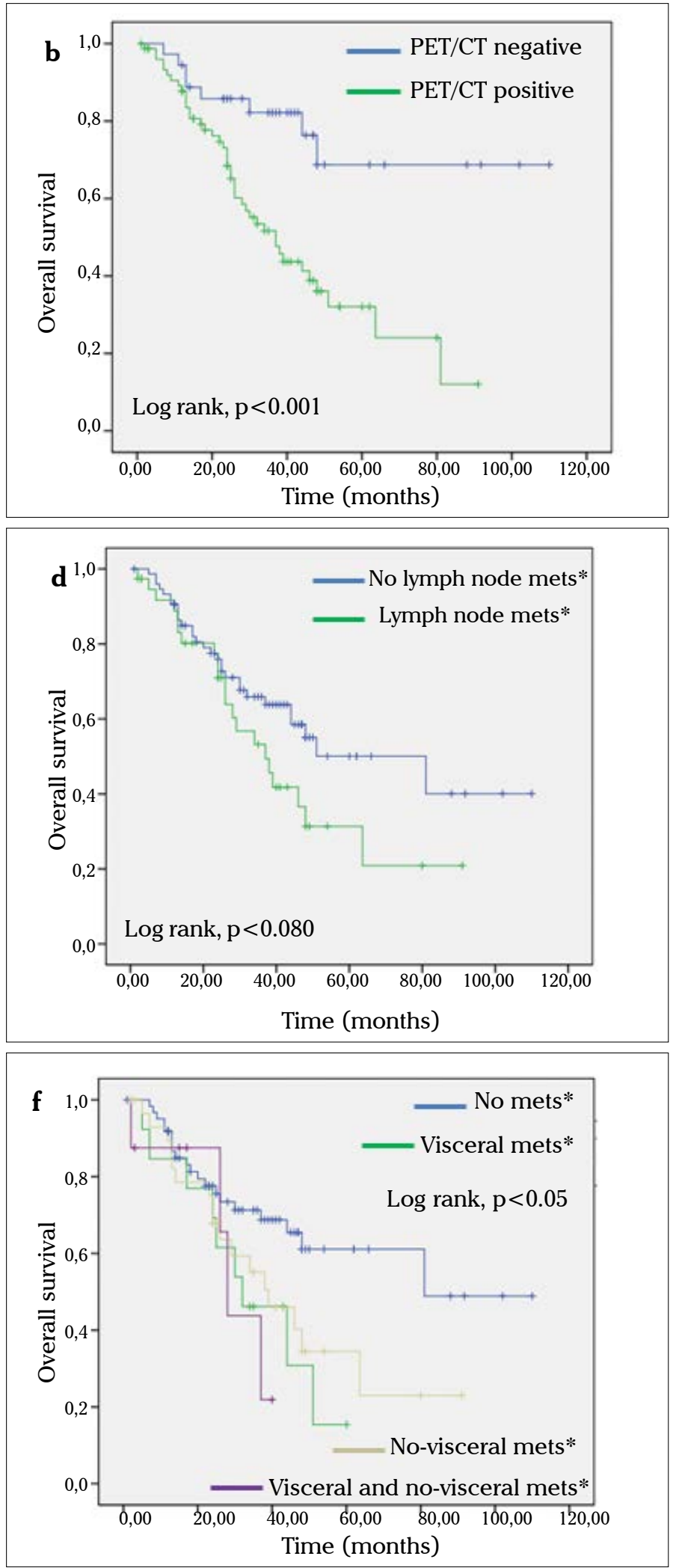

patients undergoing PET/CT for the recurrence of disease and were later confirmed by bone scan. The value of CA125 was 
Table 5. Univariate and multivariate Cox regression analysis

\begin{tabular}{|c|c|c|c|c|c|c|}
\hline & \multicolumn{2}{|c|}{ Univariate analysis } & \multicolumn{4}{|c|}{ Multivariate analysis } \\
\hline & HR & CI $95 \%$ & $\mathbf{p}$ & HR & CI $95 \%$ & $\mathbf{p}$ \\
\hline Age & 1.042 & $1.018-1.066$ & $<0.001$ & 1.040 & $1.014-1.067$ & $<0.005$ \\
\hline Histology & 0.890 & $0.783-1.011$ & 0.074 & 1.023 & $0.910-1.150$ & 0.760 \\
\hline FIGO staging & 1.200 & $0.944-1.525$ & 0.137 & - & - & - \\
\hline Grade & 1.174 & $0.836-1.647$ & 0.355 & - & - & - \\
\hline CA125 & 2.780 & $1.517-5.097$ & $<0.005$ & 1.942 & $0.957-3.941$ & 0.066 \\
\hline PET findings & 3.361 & $1.571-7.191$ & $<0.005$ & 1.117 & $0.346-3.605$ & 0.853 \\
\hline Peritoneum recurrence at PET & 2.708 & $1.538-4.770$ & $<0.005$ & 2.408 & $1.051-5.517$ & $<0.05$ \\
\hline Local and distant metastasis at PET & 1.640 & $0.934-2.879$ & 0.085 & 0.471 & $0.086-2.567$ & 0.384 \\
\hline Liver metastasis at PET & 1.704 & $0.820-3.540$ & 0.153 & - & - & - \\
\hline Lung metastasis at PET & 2.381 & $0.942-6.021$ & 0.067 & 1.947 & $0.503-7.539$ & 0.335 \\
\hline Bone metastasis at PET & 1.803 & $0.645-5.036$ & 0.261 & - & - & - \\
\hline Visceral/non visceral metastasis at PET & 1.369 & $1.053-1.780$ & 0.019 & 1.513 & $0.666-3.437$ & 0.323 \\
\hline
\end{tabular}

consistently different among patients with visceral, non-visceral, and mixed disease (ANOVA, $\mathrm{p}<0.005$ ). Similarly, a high value of CA125 was associated with diffuse peritoneal dissemination at PET/CT; however, this result was not statistically significant (value: $300.4 \pm 849.8$ vs. $151.8 \pm 368.23 \mathrm{UI} / \mathrm{mL}$; Student's t-test; $\mathrm{p}=0.218$ ). For positive PET/CT scans, the SUVmax of the hottest lesion was recorded. No differences for the mean SUVmax were found among histological types (ANOVA test; $p=0.558$ ), stages (ANOVA test; $\mathrm{p}=0.463$ ), and grading (ANOVA test; $p=0.780$ ). We found that a SUVmax cutoff of 4.0 accurately indicated the presence of disease in our cohort of patients (sensitivity: 96\% and specificity: 74\%; AUC: 0.843, p<0.005). In patients with confirmed peritoneal carcinomatosis, the median SUVmax was 10.17 (range: 3.53 to 21), while SUVmax values of 8.75 (3.54 to 16.9 ), 5.5 (3.5 to 18.0 ), and 11.3 (4.5 to 26.6 ) were found in patients with non-visceral (lymph node and bone), visceral (liver and lung), and mixed (visceral and no-visceral) metastases, respectively. No correlation between SUVmax and CA125 was found $\left(R^{2}=0.006 ; p=N S\right)$.

\section{PET/CT, CA125 and prognosis}

The results obtained by Cox regression analysis are shown in Table 5. As illustrated by univariate analysis, older age, high CA125 level, positive PET results, and peritoneum recurrence determined by PET were correlated with an unfavorable prognosis. Conversely, by multivariate regression analysis, only high age and the presence of peritoneum recurrence at PET were identified as independent predictors of unfavorable prognosis. The survival analysis demonstrated that GS was significantly better in patients who had negative CA125 values at the time of PET/CT imaging and a negative PET/CT scan, and in patients with no evidence of peritoneum recurrence and distant metastases by PET (Figure 3 a-e). Moreover, a negative PET/CT was associated with a more favorable prognosis, also in the case of normal CA125 levels (survival rates after 48 months were 72.8 and $49.4 \%$ for negative and positive PET/ CT results, respectively). Finally, patients with non-visceral metastases, such as bone and lymph nodes, observed by PET had more favorable prognoses compared to those with visceral metastases (GS at 60 months: $24 \%$ vs. 16\%, respectively).

\section{Discussion}

In accordance with our findings, FDG PET/CT has a higher predictive value than the CA125 serum marker in the detection of disease recurrence. Indeed, in our population, 63 patients had serum CA125 levels of $35 \mathrm{UI} / \mathrm{mL}$ or less; however, at least $35 \%$ of them showed a true-positive PET/CT result. Bhosale et al. (8) previously demonstrated that FDG PET/CT is able to detect OC recurrence in patients with normal CA125 levels, showing higher sensitivity than CT. Additionally, we found that a CA125 cut-off of $32.5 \mathrm{UI} / \mathrm{L}$, slightly lower than the limit of normal levels (35 UI/mL), was useful to predict a positive PET/CT scan; this result agrees with other studies in which PET/CT confirmed the presence of early disease relapse, even in patients with a low level of serum CA125 (4). In our analysis, more than $60 \%$ of scans were performed secondary to clinical and conventional imaging when recurrence was suspected, implying that physicians find this imaging modality helpful in guiding their therapeutic choices. FDG PET/CT modified management in approximately half of the studied patients, resulting in the initiation of previously unplanned treatment or changes in previously defined therapeutic approaches. This result is in line with the current data in the literature (9-12). The evaluation of disease recurrence in patients with $\mathrm{OC}$ is often performed by imaging modalities because of the extension of the disease. In fact, obtaining histopathologic confirmation is not very common, because the patients are mostly treated with chemotherapy. On the other hand, patients with a positive PET/CT and high CA125 levels had a recurrence of disease more frequently, and therefore 
did not require any verification; however, it was necessary to treat them as soon as possible. PET/CT yielded false negative results in patients with low-grade tumors, such as borderline tumors with peritoneal microimplants and in clear cell OC. Moreover, small areas where the dissemination of a small number of tumors with nodules smaller than $5 \mathrm{~mm}$ occurs can cause false negative results (13). Small implants $(<5 \mathrm{~mm})$ and low disease volumes are inconsistently recognized, particularly in upper abdomen quadrants including the hepatic dome, epigastrium, and left upper abdomen. These areas commonly experience miliary spread of carcinomatosis, which is difficult to visualize with the reduced spatial resolution of PET scanners. Furthermore, false positive PET/CT results can be caused by inflammatory and infectious processes, particularly after treatment. In our cohort of patients, PET/CT produced false positive findings in 7 cases: in 4 cases due to chronic abdomen inflammation, in 1 case secondary to bronchitis, in 1 case due to abnormal liver uptake, and in the final case due to an adrenal functional adenoma. Despite these pitfalls, PET/CT has proved to be superior to CT and magnetic resonance imaging for the depiction of recurrent disease (14). In accordance with our results, we found that the inclusion of PET/CT in the management of patients with recurrent ovary cancer had an impact on the decision making in $42 \%$ of subjects. As reported in the literature, the change in management of patients with OC who have undergone FDG PET/ CT ranges between $25 \%$ and $58 \%(10,15-18)$.

OC can disseminate to the peritoneum. Knowledge of disease mapping in the abdomen can be useful to determine optimal surgical cytoreduction, which predicts patient outcome. Anatomical imaging can fail to determine the real extent of metastatic disease and intra-abdominal tumor deposits. In our study, PET/CT was highly diagnostic in defining the extension of metastatic disease, including metastasis in normal-sized pelvic retroperitoneal lymph nodes and in unsuspected distant sites, providing a low rate of false negative findings. As previously stated in the literature, PET/CT can show metastatic implants in the peritoneum as focal or diffuse areas of uptake, distributed on serosal and peritoneal surfaces; sensitivity ranging between 58 and $100 \%$ has been reported $(15,19)$. Similarly, a high value of CA125 was associated with diffuse peritoneal dissemination; however, this result was not statistically significant. No evidence of a relation between the anatomical sites of tumors and serum CA125 levels was found; also, no correlations were identified between histological type, grading of disease, and CA125 biomarker level.

Less data are currently available regarding the correlation between prognosis and the value of PET/CT in OC. Nakamura et al. (20) and Chung et al. (18) investigated the relationship between PET/CT findings and prognosis before any treatments. The authors found that preoperative CA125 levels, metabolic tumorvolume, total lesion glycolysis, and SUVmax were statistically significant independent prognostic factors for progression-free survival in patients with OC. Also, Risum et al. (21) evaluated the link between prognosis and preoperative PET/CT findings in patients with advanced OC, demonstrating that the median overall survival (OS) of PET/CT stage III and stage IV were 30.5 and 29.9 months, respectively. Avril et al. (22) demonstrated that OC patients responding to chemotherapy showed a longer median OS than non-responders. Only three studies evaluated the prognostic role of PET (7) and PET/CT $(23,24)$ in patients with OC after primary treatment. The results by Kurosaki et al. (7) are similar to the present report, showing that the serum CA125 level significantly correlated with the prognosis. However, in contrast to the study by Kurosaki et al. (7), we also found that a positive PET/CT finding and the presence of peritoneal implantations at PET are significantly associated with a shorter GS. Moreover, we determined by multivariate analysis that the presence of peritoneal carcinomatosis as determined by PET is an independent prognostic variable of an unfavorable prognosis. Therefore, the evidence of peritoneum invasion should be carefully addressed during the PET/CT examination, as it provides strong independent prognostic data.

The most important limitation of the present study is the retrospective nature of the analysis. Nevertheless, the results obtained are in line with those presented by other studies. Whether PET/CT should be routinely used instead of using a combination of conventional imaging modalities needs to be evaluated.

In conclusion, FDG PET/CT has utility in patients when OC relapse is suspected, particularly when CA125 levels are elevated. However, PET/CT can be employed also in patients with clinical symptoms of suspected cancer relapse without increased levels of this tumor marker. Meanwhile, both an abnormal CA125 value and a positive PET/CT scan are related to a poor GS. Finally, the presence of peritoneum recurrence as determined by PET is independently associated with a shorter prognosis after a period ranging between 11 and 50 months. Additional prospective trials designed with a large number of cases and examining the diagnostic and prognostic meaning of FDG PET/CT in the early detection of $\mathrm{OC}$ recurrence independent of CA125 levels are recommended.

Ethics Committee Approval: Ethics committee approval was not received due to the nature of the study.

Informed Consent: Written informed consent was obtained from patients who participated in this study.

Peer-review: Externally peer-reviewed.

Author Contributions: Concept - L.E., M.D.P., V.Z.; Design - L.E., M.D.P.; Supervision - V.Z., M.G., M.N.; Resource - M.O.N., G.B.N.; Materials - A.R.; Data Collection and/or Processing - L.E., M.D.P.; Analysis and/or Interpretation L.E., M.D.P.; Literature Search - O.N., A.R., G.B.N.; Writing - L.E., M.D.P.; Critical Reviews - V.Z., M.N., M.G.

Conflict of Interest: No conflict of interest was declared by the authors.

Financial Disclosure: The authors declared that this study has received no financial support.

\section{References}

1. Cannistra SA. Cancer of the Ovary. N Engl J Med 2004; 351: 2519-29. [CrossRef]

2. Herzog TJ. Recurrent Ovarian Cancer: How Important Is It to Treat to Disease Progression? Clin Cancer Res 2004; 10: 7439-49. [CrossRef]

3. Rustin GJ, Nelstrop AE, McClean P, Brady MF, McGuire WP, Hoskins WJ, et al. Defining Response of Ovarian Carcinoma to Initial Chemotherapy According to Serum Ca 125. J Clin Oncol 1996; 14: 1545-51. 
4. Rustin GJ, Nelstrop AE, Tuxen MK, Lambert HE. Defining Progression of Ovarian Carcinoma During Follow-up According to Ca 125: A North Thames Ovary Group Study. Ann Oncol 1996; 7: 361-4. [CrossRef]

5. Peng NJ, Liou WS, Liu RS, Hu C, Tsay DG, Liu CB. Early Detection of Recurrent Ovarian Cancer in Patients with Low-Level Increases in Serum Ca-125 Levels by 2-[F-18]Fluoro-2-Deoxy-D-Glucose-Positron Emission Tomography/Computed Tomography. Cancer Biother Radiopharm 2011; 26: 175-81. [CrossRef]

6. Santillan A, Garg R, Zahurak ML, Gardner GJ, Giuntoli RL 2nd, Armstrong DK, Bristow RE. Risk of Epithelial Ovarian Cancer Recurrence in Patients with Rising Serum Ca-125 Levels within the Normal Range. J Clin Oncol 2005; 23: 9338-43. [CrossRef]

7. Kurosaki H, Oriuchi N, Okazaki A, Tamaki T, Uki A, Izuta M, et al. Prognostic value of FDG-PET in patients with ovarian carcinoma following surgical treatment. Ann Nucl Med 2006; 20: 171-4. [CrossRef]

8. Bhosale P, Peungjesada S, Wei W, Levenback CF, Schmeler K, Rohren E, et al. Clinical utility of positron emission tomography/computed tomography in the evaluation of suspected recurrent ovarian cancer in the setting of normal CA-125 levels. Int J Gyn Cancer 2010; 20: 936-44. [CrossRef]

9. Fulham MJ, Carter J, Baldey A, Hicks RJ, Ramshaw JE, Gibson M. The Impact of PET/CT in Suspected Recurrent Ovarian Cancer: A Prospective Multi-Centre Study as Part of the Australian Pet Data Collection Project. Gynecol Oncol 2009; 12: 462-8. [CrossRef]

10. Simcock B, Neesham D, Quinn M, Drummond E, Milner A, Hicks RJ. The Impact of PET/CT in the Management of Recurrent Ovarian Cancer. Gynecol Oncol 2006; 103: 271-6. [CrossRef]

11. Yuan Y1, Gu ZX, Tao XF, Liu SY. Computer Tomography, Magnetic Resonance Imaging, and Positron Emission Tomography or Positron Emission Tomography/Computer Tomography for Detection of Metastatic Lymph Nodes in Patients with Ovarian Cancer: A MetaAnalysis. Eur J Radiol 2012; 81: 1002-6. [CrossRef]

12. Soussan M, Wartski M, Cherel P, Fourme E, Goupil A, Le Stanc E, et al. Impact of FDG PET/CT Imaging on the Decision Making in the Biologic Suspicion of Ovarian Carcinoma Recurrence. Gynecol Oncol 2008; 108: 160-5. [CrossRef]

13. Sironi S, Messa C, Mangili G, Zangheri B, Aletti G, Garavaglia E, et al. Integrated FDG PET/CT in Patients with Persistent Ovarian Cancer: Correlation with Histologic Findings. Radiology 2004; 233: 433-40. [CrossRef]

14. Son H, Khan SM, Rahaman J, Cameron KL, Prasad-Hayes M, Chuang L, et al. Role of FDG PET/CT in Staging of Recurrent Ovarian Cancer. Radiographics 2011; 31: 569-83. [CrossRef]
15. Kitajima K, Murakami K, Yamasaki E, Kaji Y, Fukasawa I, Inaba N, Sugimura K. Diagnostic Accuracy of Integrated FDG PET/ContrastEnhanced CT in Staging Ovarian Cancer: Comparison with Enhanced CT Eur J Nucl Med Mol Imaging 2008; 35: 1912-20. [CrossRef]

16. Mangili G, Picchio M, Sironi S, Viganò R, Rabaiotti E, Bornaghi D, et al. Integrated PET/CT as a first-line re-staging modality in patients with suspected recurrence of ovarian cancer. Eur J Nucl Med Mol Imaging 2007; 34: 658-66. [CrossRef]

17. Bilici A, Ustaalioglu ABO, Seker M, Canpolat N, Tekinsoy B, Salepci T, Gumus M. Clinical value of FDG PET/CT in the diagnosis of suspected recurrent ovarian cancer: is there an impact of FDG PET/ CT on patient management? Eur J Nucl Med Mol Imaging 2010; 37: 1259-69. [CrossRef]

18. Chung HH, Kwon HW, Kang KW, Park NH, Song YS, Chung JK, et al. Prognostic value of preoperative metabolic tumor volume and total lesion glycolysis in patients with epithelial ovarian cancer. Ann Surg Oncol 2012; 19: 1966-72. [CrossRef]

19. Dirisamer A, Schima W, Heinisch M, Weber M, Lehner HP, Haller J, Langsteger W. Detection of Histologically Proven Peritoneal Carcinomatosis with Fused 18f-FDG PET/MDCT Eur J Radiol 2009; 69: 536-41. [CrossRef]

20. Nakamura K, Hongo A, Kodama J, Hiramatsu Y. The pretreatment of maximum standardized uptake values (SUVmax) of the primary tumor is predictor for poor prognosis for patients with epithelial ovarian cancer. Acta Med Okayama 2012; 66: 53-60.

21. Risum S, Hogdall C, Loft A, Berthelsen AK, Høgdall E, Nedergaard L, et al. The Diagnostic Value of PET/CT for Primary Ovarian Cancer--a Prospective Study. Gynecol Oncol 2007; 105: 145-9. [CrossRef]

22. Avril N, Sassen S, Schmalfeldt B, Naehrig J, Rutke S, Weber WA, et al. Prediction of Response to Neoadjuvant Chemotherapy by Sequential F-18-Fluorodeoxyglucose Positron Emission Tomography in Patients with Advanced-Stage Ovarian Cancer. J Clin Oncol 2005; 23: 7445-52. [CrossRef]

23. Liao S, Lan X, Cao G, Yuan H, Zhang Y. Prognostic predictive value of total lesion glycolysis from 18F-FDG PET/CT in post-surgical patients with epithelial ovarian cancer. Clin Nucl Med 2013; 38: 715-20. [CrossRef]

24. Sala E, Kataoka M, Pandit-Taskar N, Ishill N, Mironov S, Moskowitz CS, et al. Recurrent Ovarian Cancer: use of contrast-enhanced CT and PET/CT to accurately localize tumor recurrence and to predict patients' survival. Radiology 2010; 257: 125-34. [CrossRef] 